\title{
Perfil epidemiológico dos casos notificados de sífilis congênita no estado do Acre nos anos de 2009-2018
}

\author{
Epidemiological profile of notified cases of congenital syphilis in the state of Acre in the \\ years 2009-2018
}

\section{Perfil epidemiológico de casos notificados de sífilis congénita en el estado de Acre en los años 2009-2018}

Thaís Jardim Teodoro Branco ${ }^{1 *}$, Elaine Azevedo Soares Leal ${ }^{2}$, Thatyana Frias Freitas ${ }^{1}$, Bárbara Bueno Manzati ${ }^{1}$.

\section{RESUMO}

Objetivo: Avaliar o perfil epidemiológico dos casos notificados de Sífilis Congênita no estado do Acre nos anos de 2009-2018. Métodos: Trata-se de um estudo epidemiológico retrospectivo, de caráter descritivo e abordagem quantitativa dos casos de SC notificados no estado do Acre, no período de 2009 a 2018, coletados no Sistema de Informação de Agravos de Notificação (SINAN) e tabulados no Microsoft Excel versão 2013. Resultados: Obteve-se um aumento dos casos de SC no período analisado, onde a maior notificação ocorreu em Rio Branco. O diagnóstico foi predominantemente realizado até o $6^{0}$ dia de vida, mais frequentes em pardos, na zona urbana, com SC recente e $93 \%$ com desfecho favorável. Em relação ao perfil materno a maioria possui de 20 a 29 anos, com baixa escolaridade, $79,9 \%$ realizaram pré-natal e $42,1 \%$ só foram diagnosticadas no momento do parto, 69,8\% tiveram o tratamento inadequado, e a grande maioria dos parceiros não realizaram nenhum tratamento. Conclusão: A sífilis congênita permanece um problema de saúde pública. No presente estudo foi diagnosticado um aumento desta enfermidade ao longo dos anos, o que fomenta a necessidade da introdução de políticas públicas gravídico-puerperal para que tenha uma redução ou mesmo a extinção de casos.

Palavras-chave: Sífilis congênita, Transmissão vertical de doença infecciosa, Epidemiologia.

\section{ABSTRACT}

Objective: To evaluate the epidemiological profile of notified cases of Congenital Syphilis in the state of Acre in the years 2009-2018. Methods: This is a retrospective epidemiological study, of a descriptive nature and quantitative approach of cases of SC notified in the state of Acre, in the period from 2009 to 2018, collected in the Information System for Notifiable Diseases (SINAN) and tabulated at Microsoft Excel version 2013. Results: There was an increase in cases of SC in the period analyzed, where the highest notification occurred in Rio Branco. The diagnosis was predominantly made until the 60th day of life, more frequent in browns, in the urban area, with recent SC and $93 \%$ with favorable outcome. In relation to the maternal profile, the majority is between 20 and 29 years old, with low education, $79.9 \%$ performed prenatal care and $42.1 \%$ were only diagnosed at the time of the birth, $69.8 \%$ had inadequate treatment, and the vast majority of partners did not underwent any treatment. Conclusion: Congenital syphilis remains a public health problem. In the present study, an increase in this disease was diagnosed over the years, which promotes the need for the introduction of pregnancy-puerperal public policies so that there is a reduction or even the extinction of cases.

Key words: Congenital syphilis, Vertical transmission of infectious disease, Epidemiology.

\section{RESUMEN}

Objetivo: Evaluar el perfil epidemiológico de los casos notificados de sífilis congénita en el estado de Acre en los años 2009-2018. Métodos: Este es un estudio epidemiológico retrospectivo, de naturaleza descriptiva y enfoque cuantitativo de casos de SC notificados en el estado de Acre, en el período de 2009 a 2018, recopilados en el Sistema de Información para Enfermedades de Notificación (SINAN) y tabulados en Microsoft Versión Excel 2013. Resultados: Hubo un aumento en los casos de SC en el período analizado,

${ }^{1}$ Centro Universitário Uninorte (UNINORTE). Rio Branco - AC. *E-mail: thaisteodoro2@gmail.com

2 Universidade Federal do Pará (UFPA). Belém - PA. 
donde la notificación más alta ocurrió en Rio Branco. El diagnóstico se realizó predominantemente hasta el día 60 de vida, más frecuente en marrones, en el área urbana, con SC reciente y 93\% con resultado favorable. En relación con el perfil materno, la mayoría tiene entre 20 y 29 años, con baja educación, el $79.9 \%$ realizó atención prenatal y el $42.1 \%$ solo fueron diagnosticados en el momento del parto, el $69.8 \%$ tenía un tratamiento inadecuado y La gran mayoría de los compañeros no se sometieron a ningún tratamiento. Conclusión: La sífilis congénita sigue siendo un problema de salud pública. En el presente estudio, se diagnosticó un aumento de esta enfermedad a lo largo de los años, lo que promueve la necesidad de introducir políticas públicas de embarazo-puerperal para que haya una reducción o incluso la extinción de casos.

Palabras clave: Sífilis congénita, Transmisión vertical de enfermedades infecciosas, Epidemiología.

\section{INTRODUÇÃO}

A sífilis congênita (SC) é causada pela bactéria Treponema pallidum transmitida da geste infectada ao concepto por via transplacentária ou pelo contato do bebê com lesões ativas no canal de parto. A maior probabilidade de transmissão ocorre quando as gestantes se encontram nas fases primária e secundária (CAVALCANTE ANM, et al., 2019). Dessa maneira o comprometimento advindo desta infecção é variável de acordo com fatores como o tempo de exposição fetal ao treponema, a carga treponêmica materna, a virulência, o tratamento da infecção materna e a coinfeccção materna pelo vírus da imunodeficiência humana ou outras doenças que causem imunodeficiência (FRANÇA ISX, et al., 2015).

Mundialmente, a sífilis ainda afeta um número elevado de gestantes. A Organização Mundial de Saúde (OMS) presumi uma incidência de 12 milhões de novos casos de sífilis anualmente, no mundo, sendo 1 milhão em gestantes. Nos Estados Unidos, a prevalência da SC teve aumento de 27,5\% entre 2013 e 2014, chegando a 11,6 casos/100.000 nascidos vivos (NV) em 2014 (ANDRADE ALMB, et al., 2018).

No que tange ao Brasil, desde 1986 a SC foi incluída entre as doenças de notificação compulsória. Entretanto, quando observado o panorama nacional ao longo do tempo, percebe-se que pouco mudou, dado preocupante, uma vez que a sífilis é doença tratável/curável, com tratamento de baixo custo quando não há complicações (MOREIRA KFA, et al., 2017).

Em 2010, foi determinada pelo Fundo das Nações Unidas para a Infância (UNICEF) e pela Organização Pan Americana da Saúde (OPAS), uma meta para a redução da incidência de SC na América Latina para 0,5 casos/1.000 NV até 2015. Porém os dados brasileiros mais recentes, expressos no Boletim Epidemiológico de Sífilis de 2016, demonstram não só o insucesso em atingir essa meta, como também as crescentes taxas de incidência e mortalidade infantil por essa doença (ANDRADE ALMB, et al., 2018).

É importante salientar que o recurso terapêutico em gestantes sifilíticas fundamenta-se no uso da penicilina benzatina, por via parenteral, como a única droga com eficácia documentada na prevenção e tratamento da transmissão vertical. Nos anos de 2014, 2015 e 2016 ocorreu a escassez do fungo Penicilium sp, matéria prima para a produção do fármaco aludido (OLIVEIRA ALL, et al., 2020).

De modo que isso contempla um dos fatores que contribuíram para a elevação da SC, onde a incidência da mesma em menores de um ano passou de 1,7 casos/1.000 NV em 2004 para 6,5 casos/1.000 NV em 2015 (ANDRADE ALMB, et al., 2018). Nessa perspectiva, o objetivo do presente estudo é avaliar o perfil epidemiológico dos casos notificados de Sífilis Congênita no estado do Acre nos anos de 2009-2018, para que assim se possa apresentar medidas de intervenção efetivas.

\section{MÉTODOS}

Trata-se de um estudo epidemiológico retrospectivo, de caráter descritivo e com abordagem quantitativa realizado no estado do Acre. Utilizado como critério de inclusão a população composta por todos os casos de SC notificados no referido estado, ocorridos no período de 2009 a 2018, de acordo com a Classificação Internacional de Doenças (CID 10) codificada como A50 e excluídos os casos referentes aos anos pregressos a 2008 e posterior a 2018.

Os dados desta pesquisa foram coletados no Sistema de Informação de Agravos de Notificação (SINAN), disponibilizado pelo Departamento de Informática do Sistema Único de Saúde do Brasil (DATASUS), no mês no mês de março do ano de 2020. 
As variáveis analisadas foram as seguintes características referentes ao indivíduo: sexo, raça, zona de residência, faixa etária da criança, classificação do diagnóstico final e evolução. Referente à mãe as variáveis estudadas foram: faixa etária, escolaridade, realização do pré-natal, momento do diagnóstico da sífilis materna, esquema de tratamento da mãe e tratamento do parceiro. Bem como análise referente à localização da ocorrência baseada na distribuição por microrregião IBGE de residência.

A coleta e tabulação dos dados foram submetidos ao cálculo de frequências absolutas, relativas e razões de prevalência e tabulados pelo programa Microsoft Excel versão 2013 os quais são apresentados por meio de tabelas. Por se tratar de pesquisa que utiliza informações de acesso público, este estudo não foi submetido ao Comitê de Ética em Pesquisas em Seres Humanos (CEP), de acordo com a resolução 510, de 07 de abril de 2016, do Conselho Nacional de Saúde - Brasil.

\section{RESULTADOS}

Ao longo da análise de 10 anos, no período de 2009 a 2018, contabilizou-se o total de 167.772 NV no estado do Acre, com 603 casos notificados e confirmados de SC (Tabela 1).

Tabela 1 - Casos de Sífilis Congênita notificados no Estado do Acre, no período de 2009-2018.

\begin{tabular}{ll}
\hline Ano & $\mathbf{N}$ \\
\hline 2009 & 49 \\
2010 & 18 \\
2011 & 22 \\
2012 & 38 \\
2013 & 76 \\
2014 & 91 \\
2015 & 69 \\
2016 & 68 \\
2017 & 77 \\
2018 & 95 \\
\hline Total & 603
\end{tabular}

Fonte: Branco TJT, et al., 2020. Dados extraídos do Sistema de Informação de Agravos de Notificação (SINAN), 2020.

Em relação a distribuição de casos por microrregião no estado do Acre, identificou-se que a maioria dos casos foram notificados em Cruzeiro do Sul com 16,6\% e na capital do estado, Rio Branco com 60,2\% (Tabela 2).

Tabela 2 - Casos de Sífilis Congênita notificados por microrregião do IBGE no estado do Acre, no período de 2009-2018.

\begin{tabular}{lcc}
\hline Microrregião IBGE Estado do Acre & N & $\%$ \\
\hline Sena Madureira & 32 & 5,3 \\
Brasiléia & 36 & 6,0 \\
Tarauacá & 72 & 11,9 \\
Cruzeiro do Sul & 100 & 16,6 \\
Rio Branco & 363 & 60,2 \\
\hline
\end{tabular}

Fonte: Branco TJT, et al., 2020. Dados extraídos do Sistema de Informação de Agravos de Notificação (SINAN), 2020.

$\mathrm{Na}$ análise das características sociodemográficas dos casos de SC $96,2 \%$ foram diagnosticados em até 6 dias e $3,9 \%$ nos dias posteriores. No que diz respeito a razão por sexo $48,8 \%$ é masculino, $46,6 \%$ feminino e $4,6 \%$ ignorado. A raça é mais frequente em pardos com $83,7 \%$. No tocante a zona de residência $69,2 \%$ habita na área urbana, $28,4 \%$ na rural, $1,2 \%$ na periurbana e $1,3 \%$ equivale aos ignorados/ brancos (Tabela 3 ). 
Quanto as características clínicas, evidenciou-se a classificação final a ocorrência de $84,1 \%$ com sífilis congênita recente, $4 \%$ natimorto ou aborto por sífilis, $8,6 \%$ descartados e $3,3 \%$ ignorados/brancos. No que se refere evolução $93 \%$ estavam vivos no momento da notificação em contrapartida que $2,5 \%$ tiveram óbito pelo agravo, $0,2 \%$ óbito por outra causa e 4,4\% foram ignorados ou brancos (Tabela 3 ).

Tabela 3 - Características sociodemográficas e clínicas dos casos de Sífilis Congênita notificados no estado do Acre, no período de 2009-2018.

\begin{tabular}{|c|c|c|}
\hline Características & $\mathbf{N}$ & $\%$ \\
\hline \multicolumn{3}{|l|}{ Faixa Etária } \\
\hline Até 6 dias & 580 & 96,2 \\
\hline $7-27$ dias & 12 & 2,0 \\
\hline 28 dias $a<1$ ano & 10 & 1,7 \\
\hline 1 ano (12 a 23 meses) & 1 & 0,2 \\
\hline 2 a 4 anos & 0 & 0,0 \\
\hline 5 a 12 anos & 0 & 0,0 \\
\hline \multicolumn{3}{|l|}{ Sexo } \\
\hline Ignorado & 28 & 4,6 \\
\hline Masculino & 294 & 48,8 \\
\hline Feminino & 281 & 46,6 \\
\hline \multicolumn{3}{|l|}{ Raça } \\
\hline Ignorado/Branco & 40 & 6,6 \\
\hline Branca & 40 & 6,6 \\
\hline Preta & 8 & 1,3 \\
\hline Parda & 505 & 83,7 \\
\hline Indígena & 10 & 1,7 \\
\hline \multicolumn{3}{|l|}{ Zona de residência } \\
\hline Ignorado/Branco & 8 & 1,3 \\
\hline Urbana & 417 & 69,2 \\
\hline Rural & 171 & 28,4 \\
\hline Periurbana & 7 & 1,2 \\
\hline \multicolumn{3}{|l|}{ Classificação final } \\
\hline Ign/Branco & 20 & 3,3 \\
\hline Sífilis Congênita Recente & 507 & 84,1 \\
\hline Natimorto/Aborto por Sífilis & 24 & 4,0 \\
\hline Descartado & 52 & 8,6 \\
\hline \multicolumn{3}{|l|}{ Evolução } \\
\hline Ign/Branco & 23 & 4,4 \\
\hline Vivo & 489 & 93,0 \\
\hline Óbito pelo agravo notificado & 13 & 2,5 \\
\hline Óbito por outra causa & 1 & 0,2 \\
\hline
\end{tabular}

Fonte: Branco TJT, et al., 2020. Dados extraídos do Sistema de Informação de Agravos de Notificação (SINAN), 2020.

No que corresponde as características nos casos de sífilis congênita correlacionada com o perfil materno e do parceiro, a faixa etária que predomina são mães de 20 a 29 anos com 47,9\%. Em relação a escolaridade $3,5 \%$ das mães eram analfabetas, 39,5\% tinham ensino fundamental incompleto, $13,1 \%$ o ensino médio incompleto, $13,9 \%$ o ensino médio completo e $2,3 \%$ cursaram o ensino superior, sendo que apenas $1 \%$ concluiu.

A respeito do pré-natal, $79,9 \%$ fizeram o acompanhamento, enquanto18,1\% não realizaram. Das mães portadoras de sífilis que tiveram filhos com SC $41,3 \%$ foram diagnosticadas durante o pré-natal, $42,1 \%$ no momento do parto/ curetagem, $12 \%$ após o parto, $4,1 \%$ ignorado/branco e $0,5 \%$ não foi realizado o diagnóstico.

Além do que, $69,8 \%$ realizaram o tratamento de forma inadequada, $14,9 \%$ não realizaram a terapia e somente $4 \%$ realizaram tratamento de forma adequada. Quanto ao parceiro, $73,8 \%$ não realizaram tratamento e somente $17,6 \%$ ) se trataram (Tabela 4). 


\section{Revista Eletrônica Acervo Saúde / Electronic Journal Collection Health | ISSN 2178-2091}

Tabela 4 - Características sociodemográficas, clínicas e do parceiro das gestantes sororreagentes para sífilis estado do Acre, no período de $2009-2018$.

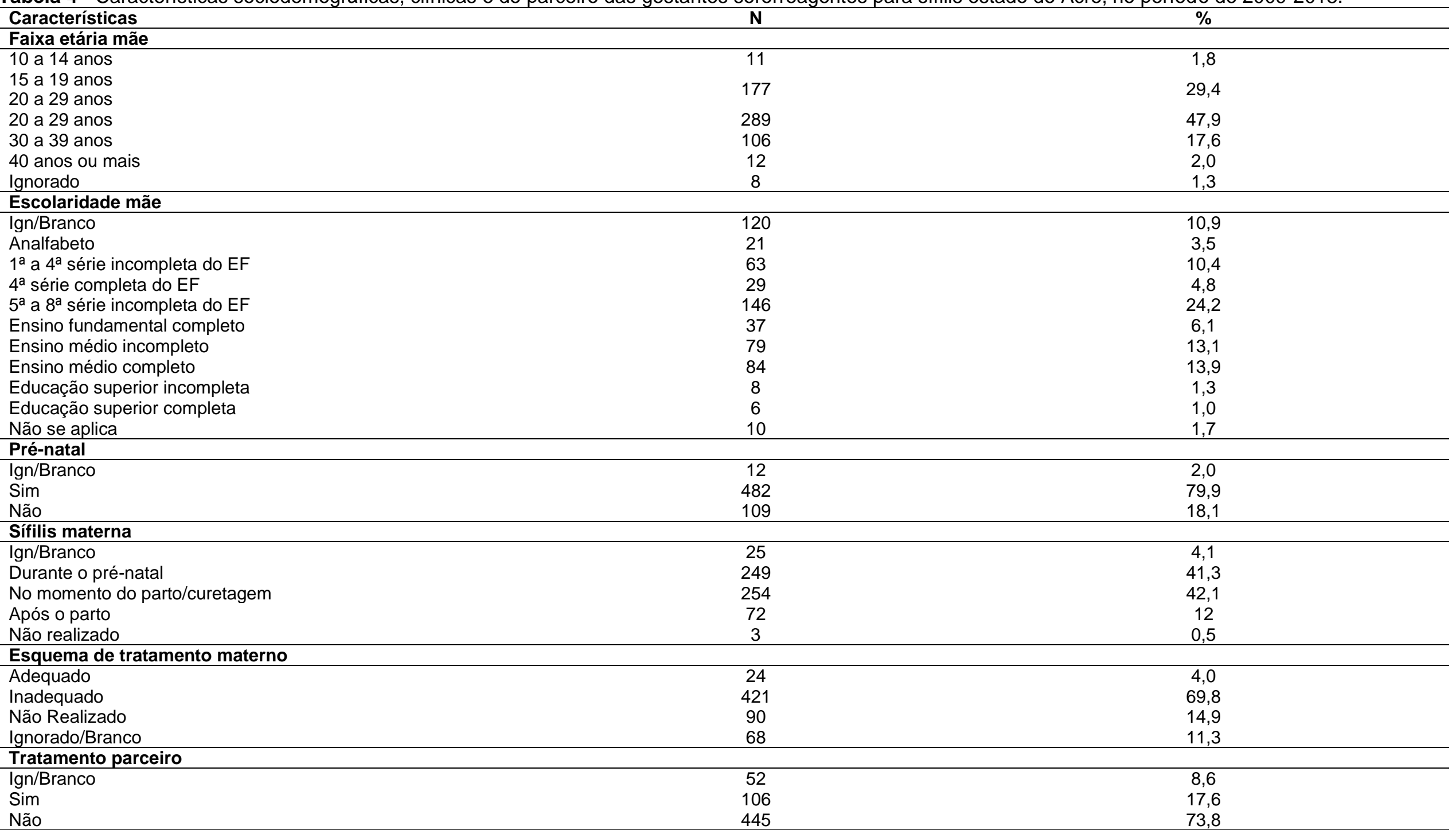

Fonte: Branco TJT, et al., 2020. Dados extraídos do Sistema de Informação de Agravos de Notificação (SINAN), 2020. 
O tratamento de escolha nos anos de 2015 a 2018 nas gestantes estudadas foi a penicilina benzatina com $95,7 \%$, sendo que $0,7 \%$ realizaram outro esquema, $2,2 \%$ (não realizaram o tratamento e $1,4 \%$ dos casos tiveram tratamento ignorado. Importante salientar que nessa pesquisa foram consideradas todas as gestantes em que foi administrado a partir de 1 dose da medicação.

Tabela 5 - Casos de gestantes com sífilis segundo esquema de tratamento prescrito por ano de diagnóstico no estado do Acre, no período de 2009-2018.

\begin{tabular}{lcccccc}
\hline Tratamento & $\mathbf{2 0 1 5}$ & $\mathbf{2 0 1 6}$ & $\mathbf{2 0 1 7}$ & $\mathbf{2 0 1 8}$ & $\mathbf{N}$ & \% \\
\hline Penicilina & 284 & 313 & 410 & 593 & 1.600 & $95,7 \%$ \\
benzatina & 3 & 3 & 1 & 4 & 11 & $0,7 \%$ \\
Outro Esquema & 10 & 7 & 5 & 15 & 37 & $2,2 \%$ \\
Não realizado & 10 & &
\end{tabular}

Fonte: Branco TJT, et al., 2020. Dados extraídos do Sistema de Informação de Agravos de Notificação (SINAN), 2020.

\section{DISCUSSÃO}

A meta da Organização Pan-Americana de Saúde (OPAS) e do Ministério da Saúde (MS) de reduzir a incidência de SC no Brasil, está longe de ser alcançada. Apesar de o Pacto pela Saúde ter como prioridade o controle das taxas de transmissão vertical da Sífilis (BRASIL, 2015), os casos de SC no estado do Acre vêm aumentando significativamente, fato este comprovado ao comparar os 49 casos notificados em 2009 com quase o dobro de casos em 2018, chegando a 95 casos. Esse resultado é similar ao estudo realizado em Palmas onde a taxa de incidência aumentou ano a ano, passando de 2,9 no ano de 2007 para 8,1 em 2014 por 1.000 NV8 (CAVALCANTE PA de M, et al., 2017).

Outro estudo realizado no estado de São Paulo mostrou um aumento linear e constante de 1,4 casos/1.000 NV, em 2007 a 5,8 casos/1.000 NV, em 2016 (MASCHIO-LIMA T, et al., 2019). Isso mostra o quanto a quantidade de casos notificados nas diferentes regiões brasileiras é superior à meta.

Estudos internacionais também observaram elevação das taxas de incidência de SC. Na Colômbia, ocorreu um crescimento de 2,15 casos em 2005 para 3,28 casos/1.000 NV em 2011. A Espanha apresentou uma elevação discreta, de 0,000 casos em 2003 a 0,223 casos/1.000 NV em 2007. Contrariamente, estudos de diferentes países relataram uma diminuição nos números de casos. Em Shenzhen, na China, a incidência reduziu de 1,15 em 2002 para 0,10 casos/1.000 nascidos em 2011.

Declínio semelhante ocorreu em Trindade, onde a incidência passou de 6,0 em 2003 para aproximadamente 0,5 casos/1.000 NV em 2009, alcançando a meta preconizada. De 2010 a 2015 o Reino Unido e o Sul da Itália apresentaram taxas menores do que 0,02 casos por mil NV. Nos Estados Unidos, a SC vem apresentando uma diminuição progressiva e em 2012 apresentou a menor quantidade de casos notificados. Sendo assim é notório que a as incidências encontradas para o Brasil está acima da meta e também acima das taxas observadas em outros países. (TEXEIRA LO, et al., 2018).

A persistência das notificações de SC identificado no presente estudo pode ser resultado da melhoria da notificação de casos ao longo dos 10 anos, devido à utilização do teste-rápido, aos avanços da vigilância epidemiológica, à capacitação dos profissionais da saúde e à ampliação da cobertura do pré-natal em decorrência da implantação das equipes da Estratégia Saúde da Família (ESF) (NUNES PS, et al., 2018).

De modo que isso é corroborado pela análise das notificações correspondentes as microrregiões do IBGE no Acre, à medida que se obtém as maiores notificações nos municípios que dispõe de melhores condições de assistência à saúde, sendo 60,2\% (363) dos casos notificados em Rio Branco, seguido de Cruzeiro do Sul com 16,6\% (100). Também é relevante destacar que em 2014 houve uma expressiva elevação do número de notificações no Acre totalizando 91 casos, visto que esse fato pode ser justificado pela falta de penicilina no mercado e o aumento da triagem da sífilis, proporcionada pela descentralização na distribuição dos testes rápidos de sífilis na Atenção Básica (NUNES PS, et al., 2018). 
Em relação a análise das características sociodemográficas dos casos de SC no Acre constata-se que $96,2 \%$ (580) dos casos foram diagnosticados até o 60 dia de vida, dado similar no estado do Paraná com $94,8 \%$, de forma a ratificar que o diagnóstico de SC ocorreu em tempo oportuno, permitindo assim a possibilidade de início do tratamento precoce (SIGNOR M, et al., 2018) (GUIMARÃES TA, et al., 2018).

No que diz respeito a razão por sexo, não há nenhum fator que evidencia a predileção da enfermidade. Em virtude que a sífilis não é uma infecção seletiva, sendo assim, qualquer sexo está vulnerável a adquirir a SC, pois o que determina a infecção transplacentária é o tratamento da mãe (SIVA LCVC, et al., 2017). A raça é mais frequente em pardos com $83,7 \%$ (585). No estado da Bahia em pesquisa realizada no período de 2007 a 2017 67,3\% eram não brancos, em contrapartida o estudo realizado no Paraná no mesmo período há um predomínio a cor branca, o que aponta que esta variável pode ser justificada em função da localidade na qual ocorreu a pesquisa, pois condiz com o processo de colonização sendo estes por africanos, alemães e italianos respectivamente, bem como a população dos devidos estados se autodeclaram (MOREIRA KFA, et al.,2017; SANTOS IN, et al., 2019).

Quanto à zona de residência houve um maior número de casos notificados na zona urbana com $69,2 \%$ (417). Resultados semelhantes foram encontrados em Sobral (Ceará) com 95\% e no Paraná com 89,47\% (MOREIRA KFA, et al., 2017; LIMA VC, et al., 2017). Esses dados refletem o grande número de pessoas vivendo na zona urbana, característica observada na maioria absoluta das cidades brasileiras. De modo geral, os estudos indicam que as infecções sexualmente transmissíveis são mais frequentes entre os moradores de zonas urbanas (MACÊDO VC, et al., 2017). Contudo, um aspecto que assume destaque é a persistência de números relevantes SC na zona rural. Tal fato evidencia que essa população deve ter acesso a políticas de prevenção e tratamento dessa doença, além de iniciativas para promover seu acesso ao pré-natal de qualidade (MARQUES JVS et al., 2018; MOROSKOSKI M, et al., 2018).

Quanto a característica clínica a sífilis congênita pode ser classificada em recente, tardia, natimorto ou aborto. SC recente ocorre quando os sinais e sintomas são observados até o segundo ano de vida, já a tardia ocorre após esse período.

Os resultados deste estudo mostraram que a maioria dos casos $84,1 \%$ (507) obteve classificação final de SC recente, semelhante ao encontrado em pesquisa publicada em 2018, realizada no estado do Maranhão (GUIMARÃES TA, et al., 2018). No tocante a evolução, o resultado apresentou-se favorável onde 93\% (489) ficaram vivos em contrapartida que 2,5\% (13) tiveram óbito pelo agravo, revelando um tratamento eficaz dos portadores de SC, ao mesmo tempo que indica a necessidade de ações de saúde para intervir em mortes de causas evitáveis (REIS GJ dos, et al., 2018).

No que tange ao panorama do perfil materno os resultados desse estudo apontam que faixa etária de 20 a 29 anos é a mais suscetível ao acometimento pela enfermidade com 47,9\% (289), semelhante aos dados encontrados na maioria dos estudos (MARQUES JVS, et al., 2018; SOUZA BS de O, et al., 2018), exemplo do estado de São Paulo que apresentou $51,30 \%$ no período de 2007 a 2016, tal faixa etária predomina por representar o pico da fase reprodutiva (BOTTURA BR, et al., 2019). Também é importante ressaltar neste estudo 29,4\% (177) dos adolescentes possuem a infecção, demonstrando o início precoce e desprotegido da vida sexual.

Segundo ponto a se destacar é a que a maioria das mães possuem baixa escolaridade, no qual $45,5 \%$ (275) cursaram o ensino fundamental incompleto ou completo. Cenário similar foi encontrado em Fortaleza onde $65,1 \%$ possuem apenas o ensino fundamental, o que corrobora com uma série de fatores limitantes no processo saúde-doença, como a capacidade restrita no conhecimento de práticas de saúde e de fatores de risco, esclarecendo um dos motivos que tornaram essas mulheres vulneráveis a contaminação por sífilis (CARDOSO ARP, et al., 2018).

Outro problema está relacionado ao déficit na política de intervenção e acompanhamento dessas gestantes, visto que apesar de 79,9\% (482) terem realizado pré-natal, 42,1\% (254) foram diagnósticas com a enfermidade no momento do parto ou da curetagem. Esse fato evidencia que, apesar do acompanhamento médico ou da enfermagem, ainda persiste, no país, o diagnóstico tardio da infecção (CARDOSO ARP, et al., 
2018; LAFETÁ KTG, et al., 2016). Soma-se a isso, a questão de que a identificação tardia da doença na gestante se relaciona ao pior prognóstico para conclusão do tratamento em tempo hábil, e consequentemente para a prevenção da transmissão vertical (SOUZA BS de O, et al., 2018; CONCEIÇÃO HN, et al., 2019).

A sífilis pode ser transmitida para o bebê a partir da $9^{a}$ semana de gestação, apesar de ser mais frequente entre a $16^{\mathrm{a}}$ e $28^{\mathrm{a}}$ semanas, reforçando assim a necessidade do diagnóstico e tratamento precoces (CARDOSO ARP, et al., 2018).

O diagnóstico de acordo com o protocolo clínico e diretrizes terapêuticas para prevenção da transmissão vertical de HIV, Sífilis e Hepatites virais recomenda que seja realizado um dos testes treponêmicos (teste rápido ou FTA-Abs ou TPHA ou EQL ou ELISA) associado a um dos testes não treponêmicos (VDRL ou RPR ou TRUST) para que assim possa descartar reação cruzada pela gravidez ou outras infecções (BRASIL, 2019).

O exame não trepanêmico mais utilizado é VDRL que, apesar de ser um exame relativamente simples e barato, requer certa logística para execução. É possível que o início tardio do pré-natal associado à demora no retorno dos resultados, esteja levando grande proporção de gestantes a não receberem o resultado do VDRL ainda durante o pré-natal e dessa forma contribuindo para que o diagnóstico ocorra no parto/curetagem (PADOVANI C, et al., 2018).

O diagnóstico materno no momento do parto proporciona a oportunidade do tratamento da mãe e do parceiro, evitando possivelmente novas intercorrências de SC. Nesse momento, o tratamento já não é mais oportuno e eficaz, capaz de evitar a transmissão da sífilis para o bebê. Todavia, estudos ressaltam a importância do diagnóstico nessa ocasião, pois apesar da perda de oportunidade de evitar a transmissão vertical, existe a possibilidade de tratar o recém-nascido evitando a sífilis congênita tardia e outras consequências graves da infecção como a neurossífilis (CARDOSO ARP, et al., 2018; PADOVANI C, et al., 2018).

Neste estudo, em relação ao tratamento materno 69,8\% (421) foi realizado de forma inadequada, que consiste em qualquer tratamento que não seja feito com a penicilina, ou que esteja incompleto ou incompatível com a fase clínica da doença, bem como se for realizado no período superior a 30 dias antes do parto, não houver documentação comprobatória do tratamento anterior, não apresentar redução nos títulos da sorologia, ou que tenha seu parceiro não tratado, inadequadamente tratado ou com o tratamento ignorado (FRANÇA ISX, et al., 2015; CONCEIÇÃO HN, et al., 2019). No município de Niterói remete a mesma realidade onde $87,7 \%$ das mulheres tiveram o tratamento inadequado (HERINGER AL, et al., 2020).

É importante recordar que nos anos de 2014 á 2016 o Brasil passou por uma situação de grave vulnerabilidade sanitária em decorrência da ausência de plantas farmoquímicas para a produção de antibióticos por fermentação e consequentemente a dependência de importações de matéria - prima, a ponto de gerar uma situação de desabastecimento de medicamentos, como foi o caso da penicilina que é o fármaco de escolha para o tratamento das gestantes com sífilis e neonatos expostos a essa patologia (RODRIGUES PHA, et al., 2018).

Em uma pesquisa que avaliou a qualidade do pré-natal e condições clínicas dos neonatos expostos a sífilis no período de julho a dezembro de 2017 realizada em Rio Branco (Acre), retrata essa ausência da medicação, no qual o esquema terapêutico adotado aos neonatos expostos à sífilis foram predominantemente a ceftriaxona, seguindo a medida alternativa utilizada pelo Ministério da Saúde publicada numa nota informativa em 2016 a falta de penicilina. É importante ressaltar que a literatura afirma não existirem estudos que comprovem a eficácia do tratamento da sífilis em gestante e da sífilis congênita com antibióticos alternativos à penicilina.

A despeito de deduzir que a repercussão à longo prazo do tratamento da sífilis congênita com a ceftriaxona na população estudada ainda é uma incógnita (PASTRO D de OT, et al., 2019). No presente estudo o tratamento de escolha utilizado pelas gestantes foi a penicilina benzatina com $97,7 \%$ (1.600), sendo que nessa pesquisa foi considerada todas as gestantes em que foi administrado a partir de 1 dose da medicação, ou seja, esse número não necessariamente reflete o tratamento de forma adequada. 
Ainda sob o viés do tratamento é imprescindível o controle de cura da gestante a fim de realizar uma avaliação, controlando assim, as possíveis reinfecções especialmente quando o parceiro não comparece para tratamento (MOURA MCLC, et al., 2019). Dados este evidenciado na pesquisa quando $73,8 \%(445)$ dos seus parceiros não realizaram tratamento, dando ênfase a magnitude do problema da sífilis gestacional e consequentemente a sífilis congênita. De forma que estes resultados reforçam a necessidade da inclusão do parceiro sexual no pré-natal para consequente redução da transmissão vertical da SC.

\section{CONCLUSÃO}

A sífilis congênita permanece um sério problema de saúde pública. No presente estudo foi diagnosticado um aumento desta enfermidade ao longo dos anos, onde maioria das gestantes infectadas possuem de 20 a 29 anos, com baixa escolaridade e mesmo que a maioria tenha sido diagnosticada durante o pré-natal, uma grande parcela só foi diagnosticada no momento do parto, aliado a isso existe ainda a prevalência do tratamento inadequado e a persistência do não tratamento dos parceiros. O que fomenta a necessidade da introdução de políticas públicas gravídico-puerperal para que enfim esse agravo tenha uma redução ou mesmo a extinção de casos.

\section{REFERÊNCIAS}

1. ANDRADE ALMB, et al. Diagnóstico tardio de sífilis congênita: uma realidade na atenção à saúde da mulher e da criança no Brasil. Revista Paulista de Pediatria, 2018; 36(3): 376-381.

2. BRASIL. Ministério da Saúde (MS). Boletim Epidemiológico de Sífilis 2015. Ministério da Saúde, Ano IV no $01,2015$.

3. BRASIL. Ministério da Saúde (MS). Protocolo Clínico e Diretrizes Terapêuticas para Prevenção da Transmissão Vertical do HIV, Sífilis e Hepatites Virais. Ministério da Saúde, 2019.

4. BOTTURA BR, et al. Perfil epidemiológico da sífilis gestacional e congênita no Brasil - período de 2007 a 2016. Arquivos Médicos dos Hospitais e da Faculdade de Ciências Médicas da Santa Casa de São Paulo, 2019; 64(2): 6975.

5. CARDOSO ARP, et al. Análise dos casos de sífilis gestacional e congênita nos anos de 2008 a 2010 em Fortaleza, Ceará, Brasil. Ciência \& Saúde Coletiva, 2018; 23(2): 563-574.

6. CAVALCANTE ANM, et al. Factors associated with inadequate follow-up of children with congenital syphilis. Revista de Saúde Pública, 2019; 53:1-10.

7. CAVALCANTE PA de M, et al. Sífilis gestacional e congênita em Palmas, Tocantins, 2007-2014. Epidemiologia e Serviços de Saúde, 2017; 26(2): 255-264.

8. CONCEIÇÃO HN da, et al. Análise epidemiológica e espacial dos casos de sífilis gestacional e congênita. Saúde em Debate, 2019; 43, n. 123, p. 1145-1158, 2019.

9. FRANÇA ISX, et al. Fatores associados à notificação da sífilis congênita: um indicador de qualidade da assistência pré-natal. Revista Rene, 2015; 16(3): 374-38.

10. GUIMARÃES TA, et al. Sífilis em gestantes e sífilis congênita no Maranhão. Arquivos de Ciências da Saúde, v. 25, n. 2, p. 24-30, 2018.

11. HERINGER AL dos S, et al. Desigualdades na tendência da sífilis congênita no município de Niterói, Brasil, 2007 a 2016. Revista Panamericana de Salud Pública, 2020; 44:1-8.

12. LAFETÁ KRG, et al. Sífilis materna e congênita, subnotificação e difícil controle. Revista Brasileira de Epidemiologia, 2018;19(1): 63-74.

13. LIMA VC, et al. Perfil epidemiológico dos casos de sífilis congênita em um município de médio porte no nordeste brasileiro. J Health Biol Sci, 2017; 5(1):56-61.

14. MACÊDO VC de, et al. Risk factors for syphilis in women: case-control study: case-control study. Revista de Saúde Pública, 2017; 51: 1-12.

15. MARQUES JVS, et al. Perfil Epidemiológico da Sífilis Gestacional: Clínica e Evolução De 2012 A 2017. Revista Sanare, 2018; 17(02): 13-20.

16. MASCHIO-LIMA T, et al. Epidemiological profile of patients with congenital and gestational syphilis in a city in the State of São Paulo, Brazil. Revista Brasileira de Saúde Materno Infantil, 2019; 19(4): 865-872.

17. MOREIRA KFA, et al. Perfil dos casos notificados de sífilis congênita. Revista Cogitare Enfermagem, 2017; 22(2):1 10.

18. MOROSKOSKI M, et al. Perfil de Gestantes Adolescentes Diagnosticadas com Sífilis em Curitiba - PR. Revista Saúde Pública,2018: 47-58.

19. MOURA MCLC, et al. Sífilis congênita no Piauí: um agravo sem controle. Brazilian Journal of Surgery and Clinical Research - BJSCR;26(3): 29-35.

20. NUNES PS, et al. Sífilis gestacional e congênita e sua relação com a cobertura da Estratégia Saúde da Família, Goiás, 2007-2014: um estudo ecológico. Epidemiologia e Serviços de Saúde, 2018; 27(4): 1-10.

21. OLIVEIRA ALL, et al. Sífilis congênita: uma questão de políticas públicas ou desabastecimento medicamentoso? Ciências da Saúde: Campo Promissor em Pesquisa, 2020: 183-191. 
22. PADOVANI C, et al. Syphilis in during pregnancy: association of maternal and perinatal characteristics in a region of southern Brazil. Revista Latino-americana de Enfermagem, 2018; 26:1-10.

23. PASTRO D de OT, et al. Prenatal quality and clinical conditions of newborns exposed to syphilis. Journal Of Human Growth And Development, 2019; 29(2): 249-256.

24. REIS GJ dos, et al. Diferenciais Intraurbanos da Sífilis Congênita: Análise Preditiva Por Bairros do Município do Rio De Janeiro, Brasil. Cadernos de Saúde, 2018; 34(9): 1-13.

25. RODRIGUES PHA, et al. A evolução recente da indústria farmacêutica brasileira nos limites da subordinação econômica. Physis: Revista de Saúde Coletiva, 2018; 28(1):1-22.

26. SANTOS IN, et al. Perfil epidemiológico de sífilis congênita no Estado da Bahia, Brasil, 2007 a 2017. Revista Uruguaya de Enfermería, 2019; 14(2): 34-43.

27. SIGNOR M, et al. Distribuição espacial e caracterização de casos de sífilis congênita. Revista de Enfermagem Ufpe On Line, 2018; 12(2): 398-406.

28. SILVA LCVG, et al. Perfil dos casos de sífilis congênita em um município do sul de Mato Grosso. Journal Health NPEPS, 2017: 380-390.

29. SOUZA BS de O, et al. Análise epidemiológica de casos notificados de sífilis. Revista da Sociedade Brasileira de Clínica Médica, 2018; 16(2): 94-98.

30. TEIXEIRA LO, et al. Tendência temporal e distribuição espacial da sífilis congênita no estado do Rio Grande do Sul entre 2001 e 2012. Ciência \& Saúde Coletiva, 2018; 23(8): 2587-2597. 